Draft VERSION 2018 NOVEMBER 6

Preprint typeset using $\mathrm{AT}_{\mathrm{E} X} \mathrm{X}$ style emulateapj v. 11/10/09

\title{
ON THE RADIAL EXTENT OF THE DWARF IRREGULAR GALAXY IC10 $10^{1}$
}

\author{
N. Sanna ${ }^{2,3}$, G. Bono ${ }^{3,4}$, P. B. Stetson ${ }^{5}$, I. Ferraro ${ }^{4}$, M. Monelli ${ }^{6}$, M. Nonino ${ }^{7}$, P. G. Prada Moroni ${ }^{8,9}$, R. $^{2}$ \\ Bresolin $^{10}$, R. Buonanno ${ }^{3,11}$, F. Caputo ${ }^{4}$, M. Cignoni ${ }^{12,13}$, S. Degl'Innocenti ${ }^{8,9}$, G. Iannicola ${ }^{4}$, N. Matsunaga ${ }^{14}$, A. \\ Pietrinferni ${ }^{15}$, M. Romaniello ${ }^{16}$, 'J. Storm ${ }^{17}$, and A. R. Walker ${ }^{18}$
}

(Dated: drafted 2018 November 6 / Received / Accepted)

Draft version 2018 November 6

\begin{abstract}
We present new deep and accurate space (Advanced Camera for Surveys - Wide Field Planetary Camera 2 at the Hubble Space Telescope) and ground-based (Suprime-Cam at Subaru Telescope, Mega-Cam at Canada-France-Hawaii Telescope) photometric and astrometric data for the Local Group dwarf irregular IC10. We confirm the significant decrease of the young stellar population when moving from the center toward the outermost regions. We find that the tidal radius of IC10 is significantly larger than previous estimates of $r_{t} \lesssim 10^{\prime}$. By using the $I, V-I$ Color Magnitude Diagram based on the Suprime-Cam data we detect sizable samples of red giant (RG) stars up to radial distances of 18-23' from the galactic center. The ratio between observed star counts (Mega-Cam data) across the tip of the RG branch and star counts predicted by Galactic models indicate a star count excess at least at a $3 \sigma$ level up to 34-42' from the center. This finding supports the hypothesis that the huge HI cloud covering more than one degree across the galaxy is associated with IC10 (Huchtmeier 1979; Cohen 1979). We also provide new estimates of the total luminosity $\left(L_{V} \sim 9 \times 10^{7} L_{\odot}, M_{V} \sim-15.1\right.$ $\mathrm{mag})$ that agrees with similar estimates available in the literature. If we restrict to the regions where rotational velocity measurements are available $\left(\mathrm{r} \approx 13^{\prime}\right)$, we find a mass-to-light ratio $\left(\sim 10 M_{\odot} / L_{\odot}\right)$ that is at least one order of magnitude larger than previous estimates. The new estimate should be cautiously treated, since it is based on a minimal fraction of the body of the galaxy.

Subject headings: galaxies: dwarf — galaxies: individual (IC10) — galaxies: stellar content — Local Group - stars: evolution
\end{abstract}

\section{INTRODUCTION}

Photometric investigations of the stellar populations in Local Group (LG) dwarf galaxies provide firm constraints on cosmological parameters and the unique opportunity to investigate galaxy formation models

1 This research used the facilities of the Canadian Astronomy Data Centre operated by the National Research Council of Canada with the support of the Canadian Space Agency. This research is based in part on data collected at Subaru Telescope, which is operated by the National Astronomical Observatory of Japan.

${ }^{2}$ University of Virginia, 530 McCormick Road 22903 Charlottesville, VA, USA; ns2as@virginia.edu

${ }^{3}$ Univ. Roma Tor Vergata, via della Ricerca Scientifica 1, 00133 Rome, Italy

${ }^{4}$ INAF-OAR, via Frascati 33, Monte Porzio Catone, Rome, Italy

5 DAO-HIA, NRC, 5071 West Saanich Road, Victoria, BC V9E 2E7, Canada

${ }^{6}$ IAC, Calle Via Lactea, E38200 La Laguna, Tenerife, Spain

7 INAF-OAT, via G.B. Tiepolo 11, 40131 Trieste, Italy

8 Univ. Pisa, Largo B. Pontecorvo 2, 56127 Pisa, Italy

9 INFN, Sez. Pisa, via E. Fermi 2, 56127 Pisa, Italy

10 Institute for Astronomy, 2680 Woodlawn Drive, Honolulu, HI 96822, USA

11 ASI-Science Data Center, ASDC c/o ESRIN, via G. Galilei, 00044 Frascati, Italy

12 Dipartimento di Astronomia, Università di Bologna, via Ranzani 1, 40127 Bologna, Italy

${ }^{13}$ INAF-OAB, via Ranzani 1, 40127 Bologna, Italy

${ }^{14}$ Institute of Astronomy, University of Tokyo, 2-21-1 Osawa, Mitaka, Tokyo 181-0015, Japan

${ }^{15}$ INAF-OACTe, via M. Maggini, 64100 Teramo, Italy

16 ESO, Karl-Schwarzschild-Str. 2, 85748 Garching bei Munchen, Germany

17 AIP, An der Sternwarte 16, D-14482 Potsdam, Germany

18 NOAO-CTIO, Casilla 603, La Serena, Chile
(Mateo 1998; Tolstov et al. 2009; Wvse 2010). In this context dwarf irregulars (dIs) play a key role, since we still lack firm empirical and theoretical constraints concerning their evolution and the possible transition into dwarf spheroidal galaxies (Bekki 2008; Woo et al. 2008; Kormendy et al. 2009). Although the number of dwarf galaxies known in the LG is rapidly growing in the last few years, current statistics indicate that the dIs are at least one quarter of LG galaxies (McConnachie et al. 2008; Sanna et al. 2009).

Among the dIs of the LG, IC10 is an interesting system, since it underwent strong star formation activity during the last half billion years and it is considered the only LG analog of starburst galaxies. Even though IC10 has been the subject of several investigations ranging from the radio (Wilcots \& Miller 1998) to the near-infrared (NIR, Vacca et al. 2007), to the UV (Hunter 2001; Richer et al. 2001), and to the X-ray (Wang et al. 2005), its structural parameters and in particular its radial extent are poorly defined. Massey \& Armandroff (1995) found that the major axis of IC10 is $\sim 7^{\prime}$. A similar diameter $\left(\sim 6^{\prime}\right)$ was found by Jarrett et al. (2003) using the isophotal radii from 2MASS NIR images. More recently Tikhonov \& Galazutdinova (2009), using both ground-based and space images, suggested that the extent of the thick disk along the minor axis is $\approx 10.5^{\prime}$. It has also been suggested by Demers et al. (2004), using asymptotic giant branch and red giant branch (RGB) stars, that IC10 should have a halo of $\sim 30^{\prime}$ diameter. On the other hand, radio measurements by Huchtmeier (1979, hereinafter H79) indicated that IC10 has a huge envelope of neutral hydrogen extending over more than 
1 square degree $\left(62^{\prime} \times 80^{\prime}\right)$ across the sky.

We are also facing a significant uncertainty in the total mass of IC10. By using HI regions H79 found $M_{\text {tot }} \sim 1.8 \times 10^{9} M_{\odot}$, assuming a distance of $1 \mathrm{Mpc}$ and a Holmberg diameter of $\sim 10^{\prime}$. Also, Shostak \& Skillman (1989, hereinafter SS89), using high resolution maps of HI regions, measured an inclination of $45^{\circ}$ and a maximum in the rotation curve of $30 \mathrm{~km} \mathrm{~s}^{-1}\left(42 \mathrm{~km} \mathrm{~s}^{-1}\right.$ deprojected) and the same Holmberg diameter (deprojected angular diameter $\sim 13^{\prime}$ ), from which they found $M_{\text {tot }} \sim 1 \mathrm{x} 10^{9} M_{\odot}$. More recently, van den Bergh (2000), following H79, but assuming a smaller distance $(660 \mathrm{kpc}$, Sakai et al. 1999), found $M_{t o t} \sim 6 \times 10^{8} M_{\odot}$.

\section{PHOTOMETRIC DATA}

The Hubble Space Telescope (HST) data were collected with the Advanced Camera for Surveys (ACS, pointings $\alpha, \beta)$ and with the Wide Field Planetary Camera 2 (WFPC2, pointings $\gamma, \delta)$. Data from pointings $\alpha, \beta$ and $\gamma$ were already presented (Sanna et al. 2008, 2009). Pointing $\delta$ includes $24 F 555 W$-band and 24 $F 814 W$-band images of $500 \mathrm{~s}$ each. This pointing is located $\sim 3^{\prime} \mathrm{NE}$ of the galaxy center; it is outside the disk identified by Jarrett et al. (2003, see the red ellipse in Fig. 1) and inside the thick disk identified by Tikhonov \& Galazutdinova (2009). The groundbased data were collected with the prime focus camera (Suprime-Cam, pointing $\epsilon$ ) on the Subaru telescope and with Mega-Cam on the CFHT (pointing $\zeta$ ). Pointing $\epsilon$ (see Fig. 1) is located across the galaxy center and includes both shallow $(3 \mathrm{~V}, 3 R, 3 I ; 3 \times 60 \mathrm{~s}$ per band) and deep $(12 \mathrm{~V}, 12 \times 480 \mathrm{~s} ; 13 R, 13 \times 360 \mathrm{~s} ; 23 I, 23 \times 240 \mathrm{~s})$ images. The pointing $\zeta$ (see Fig. 1 ) is also located across the galaxy center and includes $3 \mathrm{~g}(3 \times 700 \mathrm{~s})$ and $3 \mathrm{i}$-band $(3 \times 400 \mathrm{~s})$ images.

Photometry on individual images was performed with DAOPHOT IV/ALLSTAR (Stetson 1987). The 786 ground-based images were simultaneously reduced with ALLFRAME (Stetson 1994); the same applies to the 392 space images. We ended up with a catalog including $\sim 1,200,000$ stars with at least one measurement in two different bands. The ground-based data were transformed into the Johnson-Kron-Cousins system using the standard stars provided by Landolt (1983, 1992) to calibrate local standards. The typical accuracy is 0.04 for $I$ and $0.05 \mathrm{mag}$ for the $V$ band. Some external chips of the Mega-Cam include a limited number of local standards and they were not included in the final calibrated catalog. To provide a homogeneous photometric catalog the ACS and the WFPC2 were transformed into the $V, I$ Johnson-Kron-Cousins system using prescriptions by Sirianni et al. (2005). The typical accuracy is of a few hundredths of magnitude in both $V$ and $I$. The conclusions of this investigation are not affected by the precision of the absolute zero-points.

\section{RESULTS AND DISCUSSION}

The ground-based data cover a sky area of $\sim 1^{\circ} \times 1^{\circ}$ while the high angular resolution of HST images allowed us to perform accurate photometry in the innermost crowded regions. We selected eight different regions, the region " $\mathrm{C}$ " is located across the galaxy center and includes data of pointings $\alpha(\mathrm{ACS} @ \mathrm{HST})$ and $\gamma$ (WFPC2@HST), while the region "W" is located $\sim 3^{\prime}$ from the center and includes the data of the pointing $\delta$ (WFPC2@HST). Regions "S1", "S2", "S3", and "S4" cover the corners of the Suprime-Cam data (pointing $\epsilon$ ) and are located at $\sim 18^{\prime}$ from the galaxy center, while regions "M1" and "M2" are two regions of the Mega-Cam data (pointing $\zeta$ ) located at $\sim 30^{\prime}$ from the galaxy center in the SW and NE directions, respectively. Fig. 2 shows the $I, V-I$ Color-Magnitude Diagrams (CMDs) of the selected regions. Data plotted in this figure show several interesting features.

i) The photometry based on HST data is deep and very accurate, and indeed the CMDs (regions "C" and "W") reach limiting magnitudes of $I \sim 25.5-26$ and $V \sim$ 26.5 - $27 \mathrm{mag}$. The same outcome applies to the Subaru data, and indeed the CMDs reach limiting magnitudes of $I \sim 25$ and $V \sim 26 \mathrm{mag}$. The CMDs based on CFHT data are shallower with limiting magnitudes $I \sim 22.5$ and $V \sim 24.5$ mag.

ii) Young MS stars $(18 \leq I \leq 25.5,1 \leq V-I \leq 1.5$ mag) show a strong radial gradient. Their number decreases rapidly when moving from the center to the outermost galaxy regions. A handful of them are visible in region "W", while the blue objects of region "S" might be field galaxies.

iii) The different apparent colors of RGB stars when moving from the center to the outermost regions further support the occurrence of differential reddening. We estimated the reddening of region "W" using the same approach adopted in Sanna et al. (2008). The ridgeline of the RGB in this field was adopted to estimate the reddening in the regions covered by Subaru data. The ridgeline of the contaminating blue field stars located in region "S4" was used to estimate the reddening in the regions covered by CFHT data. We found that the reddening is higher along the semi-major axis $(E(B-V)=0.78 \pm 0.10$ mag) and attains an almost constant value $(E(B-V)=$ $0.63 \pm 0.10 \mathrm{mag})$ in the regions covered by the Subaru data external to the HST data. In the regions covered by the CFHT data external to the Subaru data the reddening attains either similar $(E(B-V)=0.63 \pm 0.10 \mathrm{mag})$ or smaller $(E(B-V)=0.40 \pm 0.10 \mathrm{mag})$ values in the SW direction (Sanna et al. 2010, in preparation). The presence of a significant reddening variation up to $20^{\prime}$ from the center supports previous investigations based on the radial distribution of HI regions (SS89; Wilcots \& Miller 1998).

iv) Old RGB stars are ubiquitous and they can be easily identified in HST and Subaru CMDs. To properly identify RGB stars we also plotted an $\alpha$-enhanced isochrone (green line) of 13 Gyr at fixed metal content (total metallicity, $[\mathrm{M} / \mathrm{H}]=-0.66 \mathrm{dex}$; helium content, $\mathrm{Y}=0.251$ ) from the BaSTI data base (Pietrinferni et al. 2006 19. We also adopted the same true distance modulus $(\mu=24.60 \pm 0.15 \mathrm{mag}$, Sanna et al. 2008) and individual reddening estimates. The comparison between theory and observations further supports the evidence that stars located in the range $21 \lesssim I \lesssim 26$ and $1 \lesssim V-I \lesssim 2.5 \mathrm{mag}$ are bona fide old and intermediate-age RGB stars. The CMDs based on these data show also strong contamination by field stars (see the region "S1").

v) The CMDs based on CFHT data show the same field contamination as the Subaru data, and probably a

\footnotetext{
19 See also http://www.oa-teramo.inaf.it/BASTI
} 


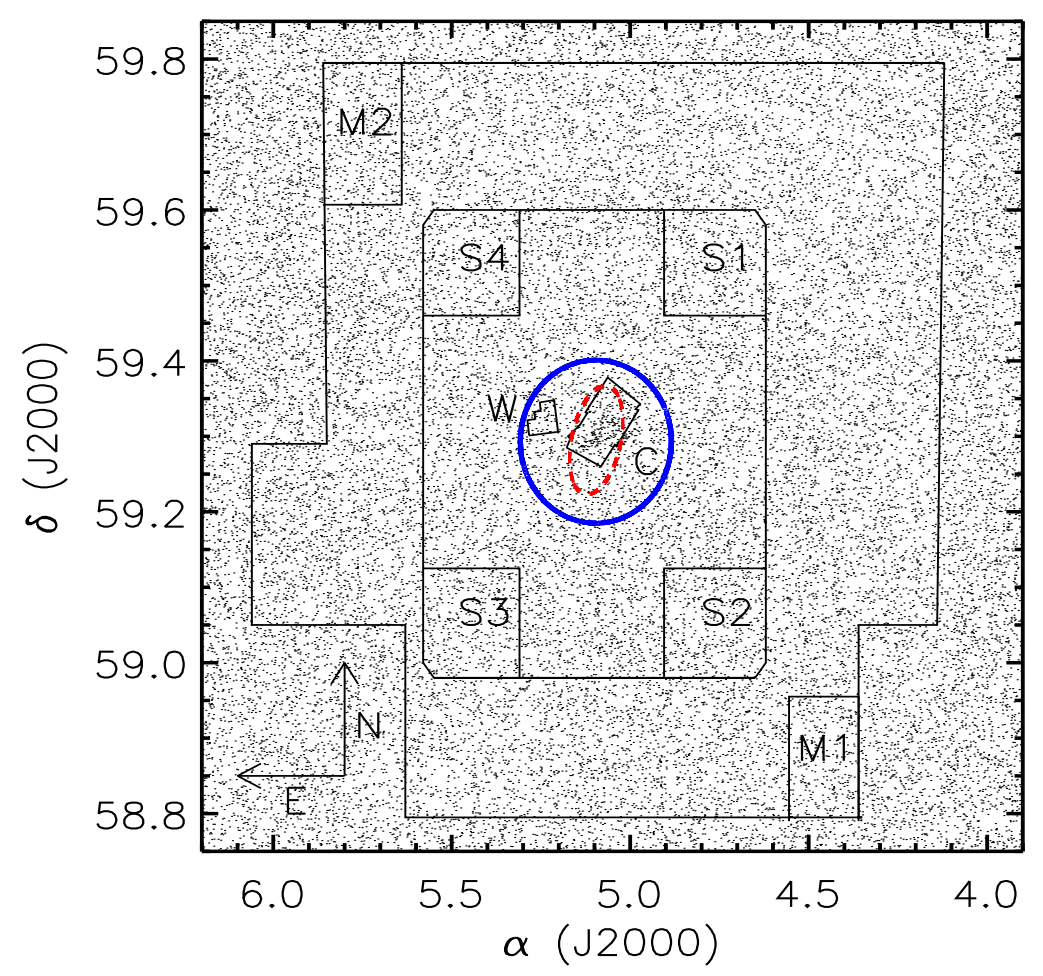

FIG. 1. - Location of ground-based and space pointings. The background is a reference image of IC10 based on randomly selected stars from the Digitized Sky Survey (DSS) catalog. The polygon labeled "C" shows the sky area covered by pointings $\alpha$ (ACS@HST) and $\gamma$ (WFPC2@HST), the polygon "W" the pointing $\delta$ (WFPC2@HST). The large rectangular polygon shows the pointing $\epsilon$ (SuprimeCam@Subaru), and the largest irregular polygon the pointing $\zeta$ (Mega-Cam@CFHT). The boxes labeled "S1", "S2", "S3", "S4" and "M1", "M2" display the regions adopted for the CMDs. The red ellipse displays the estimate of major and minor axis according to (Jarrett et al. 2003). The blue circle has a diameter of $13^{\prime}$.

small overdensity of stars in the region across the tip of the RGB (TRGB, $21 \lesssim I \lesssim 22,2 \lesssim V-I \lesssim 3.5 \mathrm{mag}$ ).

The above results indicate that the radial extent of IC10 has been significantly underestimated, and indeed according to the Subaru data the diameter is at least of the order of $36-46^{\prime}$ and probably larger than one degree according to the CFHT data.

To further constrain the radial extent of IC10, we decided to compare the observed star counts with star counts of foreground field stars predicted by Milky Way (MW) models. This approach presents several advantages when compared with the method based on the statistical subtraction of an external control field. i) It is not affected by reddening differences between the galactic and the control field. ii) It is not affected by completeness problems of the control field, thus saving telescope time. iii) The real radial extent of these stellar systems it is not known in advance. Therefore, the control fields might still be located inside their halo. The main drawback is that MW models need to be validated using deep and accurate star counts covering broad sky regions (Revle et al. 2009, and references therein).

However, the ground-based and space data sets are characterized by different limiting magnitudes. To provide a robust estimate of the completeness of the former data sets, we compared their luminosity function (LF) with the LF of the WFPC2 data for pointing "W". We adopted this approach since we are interested in esti- mating the completeness down to 1.5 magnitudes fainter than the TRGB, i.e., $I \lesssim 23$ mag. In this magnitude range the WFPC2 data are minimally affected by completeness problems. We have found that the completeness is $\sim 65 \%$ for the Subaru at the limiting magnitude of $I=23.0 \mathrm{mag}$, while it is $\sim 65 \%$ for the CFHT at the limiting magnitude of $I=22.6 \mathrm{mag}$. We have chosen the above limiting magnitudes to apply conservative completeness corrections to both the Suprime-Cam and the Mega-Cam data.

In order to compare theory and observations, we used the Pisa Castellani et al. 2002; Cignoni et al. 2006) and the Padova (Girardi et al. 2005) MW model. We focused our attention on two outer regions covering the same sky area, with the same mean reddening $(E(B-V)=0.63 \pm 0.10 \mathrm{mag})$ and located in the $\mathrm{NE}$ direction, namely the "S4" (N(stars) 4000) and "M2" $(\mathrm{N}($ stars $) \sim 3000)$ regions. The top panels of Fig. 3 show the $I, V-I$ CMD for the Pisa (left) and the Padova (right) MW models for a sky area of $1^{\circ} \times 1^{\circ}$ at the position of IC10 $\left(l=119^{\circ} ; b=-3.3^{\circ}\right)$ and assuming a reddening of $E(B-V)=0.63 \pm 0.10 \mathrm{mag}$.

The bottom panels of Fig. 3 show the ratio between the number of observed stars and the number of candidate field stars predicted by the quoted MW models. Star counts were smoothed using a Gaussian kernel at fixed $\sigma$. We also estimated the number of unresolved background galaxies, with reddened $I$-band magnitudes 


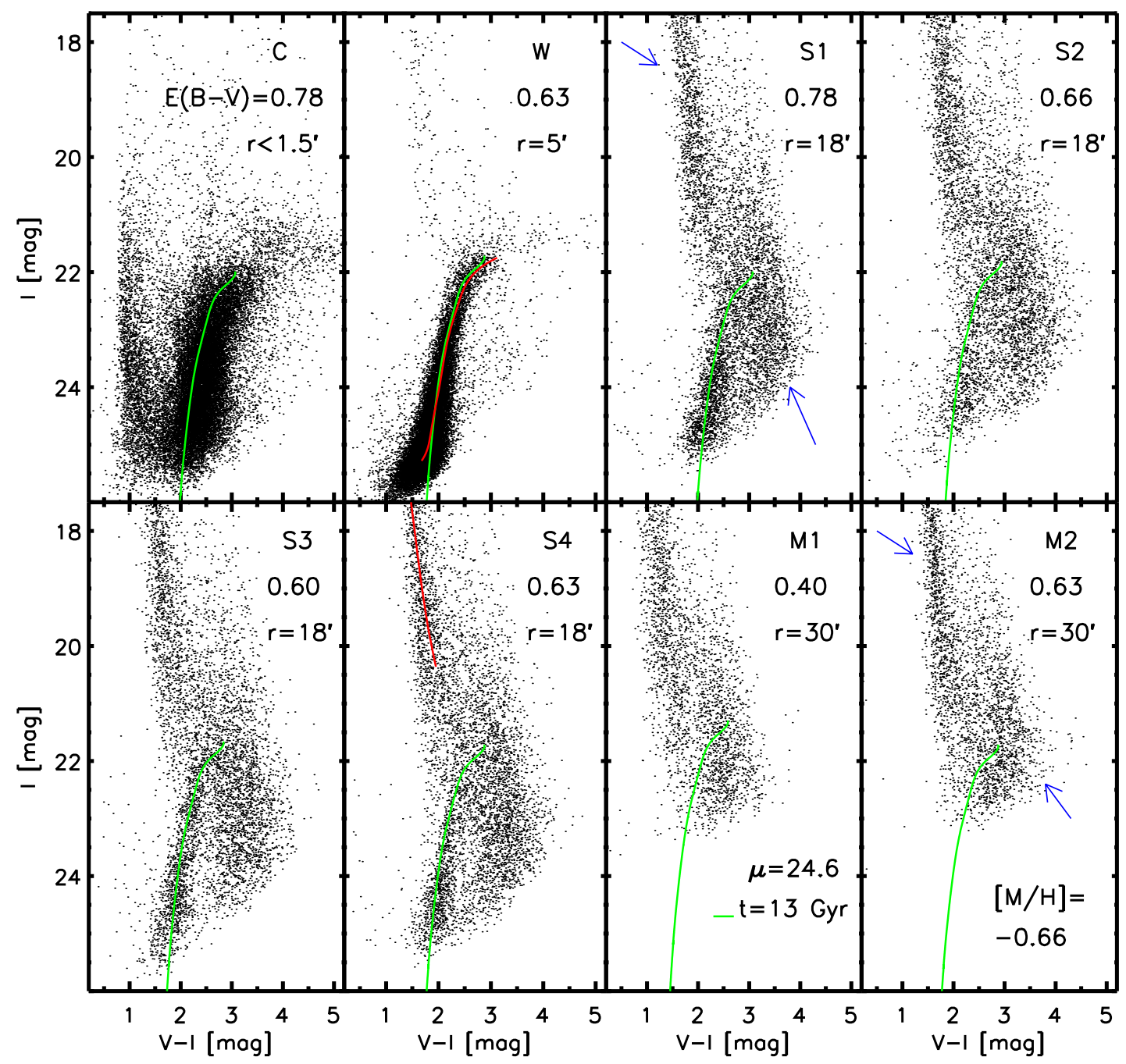

FIG. 2.- I,V-I CMDs for eight regions located at different radial distances (see labeled values and Fig. 1). Blue young, main sequence stars decrease quite rapidly when moving from the center to the outermost regions. The old RGB stars are ubiquitous in HST and in Subaru data. The reddening also changes when moving across the different regions (see labeled values). The green line shows a $\alpha$-enhanced isochrones of $13 \mathrm{Gyr}$, at fixed chemical composition $([\mathrm{M} / \mathrm{H}]=-0.66 \mathrm{dex}, \mathrm{Y}=0.25)$ from the BaSTI database. The blue arrows display the location of contaminating field stars, while the red lines plotted in panel "W" and "S4" display the ridgeline used to determine the reddening.

and $V-I$ colors typical of the tip of RGB stars, using empirical galaxy counts (Fukugita et al. 1995; Benitez 2000; Capak et al. 2004; Ferguson et al. 2004) and it was subtracted to the number of observed stars. To avoid spurious fluctuations caused by the limited sky area covered by observations, theory and observations were normalized in the bright end $(17.9 \leq \mathrm{I} \leq 18.4 \mathrm{mag})$. The bottom left panels display the comparison with the Pisa MW model. There is evidence of IC10 stars across the TRGB region ( $I=21.66 \pm 0.25 \mathrm{mag}$ ) in both the "S4" (left, $1.59 \pm 0.13$ ) and the "M2" (right, 1.38 \pm 0.12 ) field. The star count excess is at $3 \sigma$ level. The above evidence further supports the occurrence of IC10 stars in the region covered by the Mega-Cam data, since in the "S4" region we clearly identified IC10 RG stars. The bottom right panels of Fig. 3 display the comparison between obser- vations and the Padova MW model. There is once again a clear evidence of a star excess across the TRGB region in both the "S4" (left, 1.74 \pm 0.14 ) and the "M2" (right, $1.53 \pm 0.14$ ) field ( $\sim 4 \sigma$ level $)$. The two Galactic models were constructed assuming similar input parameters 20 . The difference in the star count ratios between the two models is due to the different evolutionary inputs and to the normalization of the star counts in the Solar neigh-

20 Galactic model input parameters: Kroupa initial mass function not corrected for binaries; double exponential thin disk $\left(h_{z}\right.$ (height $)=250 \mathrm{pc}, h_{R}($ scale length $)=3000 \mathrm{pc}$, constant star formation rate [SFR] for $\mathrm{t} \leq 7$ Gyr, $\mathrm{Z}$ (mean metallicity) $=0.02$ ); exponential thick disk $\left(h_{z}=1000 \mathrm{pc}, h_{R}=3500 \mathrm{pc}\right.$, constant SFR for $5 \leq \mathrm{t}<12$ Gyr, $\mathrm{Z}=0.006)$; oblate halo with $r^{1 / 4} \quad\left(h_{R}=2800\right.$ pc, $\overline{h_{q}}($ semiaxis ratio $)=0.8$, constant $\mathrm{SFR}$ for $11 \leq \mathrm{t} \leq 13$ Gyr, $\mathrm{Z}=0.0002$ ) 

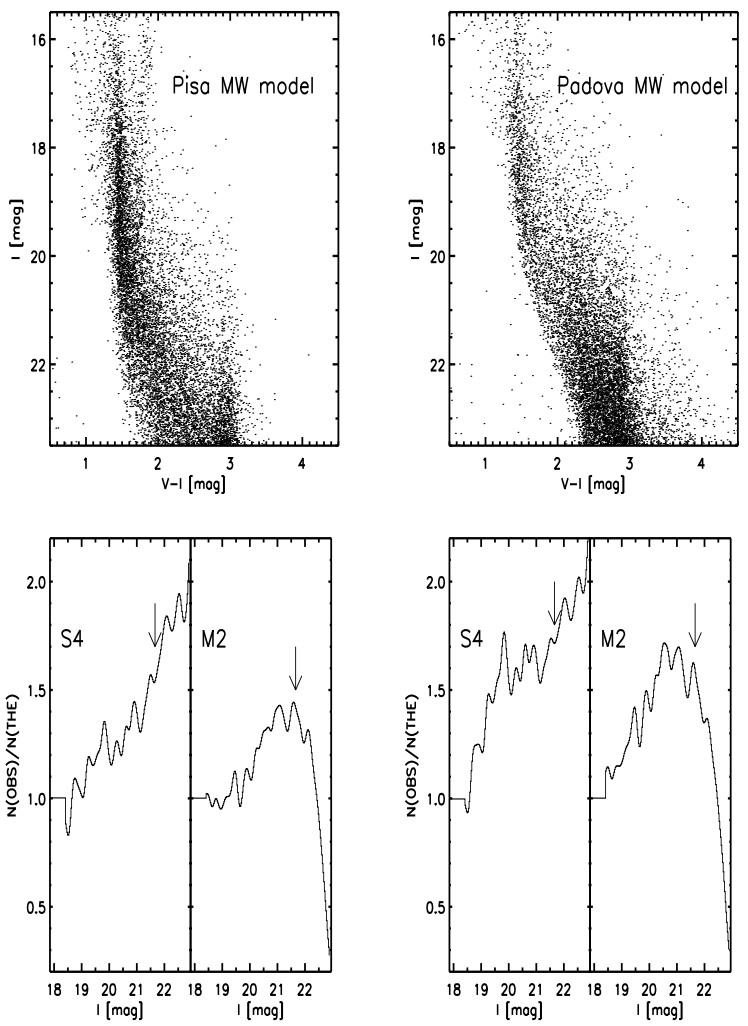

FIG. 3.- Top-Simulated CMDs for the field stars adopting a reddening of $E(B-V)=0.63 \pm 0.10 \mathrm{mag}$ in a field of view of $1^{\circ} \times 1^{\circ}$, according to the Pisa (left) and to the Padova (right) MW model. Only a small fraction of the total number of stars is plotted. Bottom-Left-Star count ratios between the stars located in the "S4" and in the "M2" region with the Pisa MW model. There is evidence of IC10 stars at the position of the TRGB, i.e. $I=$ $21.66 \pm 0.25 \mathrm{mag}$ (see the vertical arrows). Bottom-Right-Same as the left, but the ratio is between observations and the Padova MW model.

\section{bourhood.}

The above findings indicate that the radial distribution of IC10 old and intermediate-age stellar populations agrees quite well with the size of the huge hydrogen cloud detected by Huchtmeier (1979) and by Cohen (1979), and cover more than one square degree across the galaxy $\left(r \approx 34-42^{\prime}\right)$. This means that the stellar halo and the hydrogen cloud have, within the errors, similar radial extents and resolve this peculiar feature of IC10 (Tikhonov \& Galazutdinova 2009). Moreover, this evidence further supports the hypothesis that the hydrogen cloud is associated with the galaxy (stellar mass loss, pristine gas; Huchtmeier 1979; Cohen 1979; Wilcots \& Miller 1998).

To estimate the total luminosity we need to select candidate IC10 stars. To describe the procedure, Fig. 4 shows the $I, V-I$ CMDs of the stars located inside a circle of $13^{\prime}$ diameter across the galaxy center. The top panels display the photometry of space data (pointings $\alpha, \beta, \gamma$, $\delta$ ), while the bottom ones show ground data (pointing $\epsilon$, Suprime-Cam). The difference between $\epsilon 1$ and $\epsilon 2$ is in the mean reddening (see Fig. 4). For the stars located in the overlapping regions we use the HST photometry. The candidate IC10 stars were selected using different boxes in the aforementioned CMDs. The green box in- cludes young MS stars $(V-I \sim 1.5 \mathrm{mag})$, the cyan box the intermediate-age stars $(V-I \sim 3,17.5 \lesssim I \lesssim 21.5$ mag), while the pink box includes old and intermediateage RG stars $(3 \lesssim V-I \lesssim 6, I \sim 22 \mathrm{mag})$. The position of the boxes in the four CMDs was shifted according to the local reddening (see Fig. 1). The limiting magnitude of the boxes is $I=23.0$ according to the completeness experiment.

The dashed and the dashed-dotted lines plotted in the bottom right panel of Fig. 4, show two young scaled Solar abundance isochrones (Pietrinferni et al. 2004) at fixed metallicity $([\mathrm{M} / \mathrm{H}]=-0.66 \mathrm{dex})$ and ages of $t=6$ and $200 \mathrm{Myr}$, while the solid red line shows the old $\alpha$ enhanced isochrone with the same total metallicity and an age of $t=13 \mathrm{Gyr}$. These isochrones validate the position of the boxes we adopted to pinpoint the different subpopulations of IC10. The same approach was adopted to select candidate IC10 stars located between the blue circle of Fig. 1 and the outermost regions of the $\epsilon$ pointing $\left(r \lesssim 23^{\prime}\right)$. On the basis of these data and of the recent IC10 distance based on the TRGB (830 kpc, Sanna et al. 2008) we estimated a total $V$-band luminosity of $L_{V} \sim 9.13 \times 10^{7} L_{\odot}$ and a total magnitude of $M_{V}=-15.11 \mathrm{mag}$. This estimate agrees within a factor of two with similar estimates available in the literature $\left(L_{V} \sim 1.6 \times 10^{8} L_{\odot}\right.$, Mateo 1998; $M_{V}=-16.0 \mathrm{mag}$, Richer et al. 2001). The current estimate is a lower limit, since we are not including the stars located in the outermost regions covered by our photometry (pointing $\zeta)$. However, the estimates available in the literature only cover the innermost galactic regions. The difference is mainly due to the fact that the current photometry allows us a robust identification of field stars $(1 \lesssim V-I \lesssim 2$, $15 \lesssim I \lesssim 22 \mathrm{mag}$, see the blue arrow in the bottom left panel of Fig. 4). If they are even partially included, these objects introduce a systematic bias in the estimate of the total luminosity. The different assumptions concerning the adopted distance and reddening variation also help to explain the above difference.

To estimate the mass-to-light $(\mathrm{M} / \mathrm{L})$ ratio of IC10 we restricted ourself to the galactic regions where rotational velocity measurements are available (see the blue circle with a diameter of $13^{\prime}$ in Fig. 1). The luminosity inside this area is $L_{V} \sim 5.88 \times 10^{7} L_{\odot}\left(M_{V}=-14.63 \mathrm{mag}\right)$. By using the quoted true distance and diameter together with the rotation velocity based on HI regions (SS89) we found, following Huchtmeier \& Richter (2001) and Casertano \& Shostak (1980), a total mass of $M_{t o t} \sim$ $6.2 \times 10^{8} M_{\odot}$ that agrees quite well with similar estimates available in the literature (Huchtmeier 1979; van den Bergh 2000; Woo et al. 2008). Eventually, we found $\mathrm{M} / \mathrm{L} \sim 10 M_{\odot} / L_{\odot}$. Although this estimate is hampered by several empirical limitations it is at least one order of magnitude larger than the value recently provided by Woo et al. (2008). The quoted authors use two independent methods to estimate the $\mathrm{M} / \mathrm{L}$ ratios of LG dwarf galaxies: colors and inferred star formation history. They found that the median M/L ratio of dIs based on the latter approach is slightly smaller than on the former one (0.7 vs 0.8, see their Table 2$)$. However, the difference needs to be investigated in more detail, particularly in view of the severe limitations affecting the estimates of the rotational velocity and of the total 


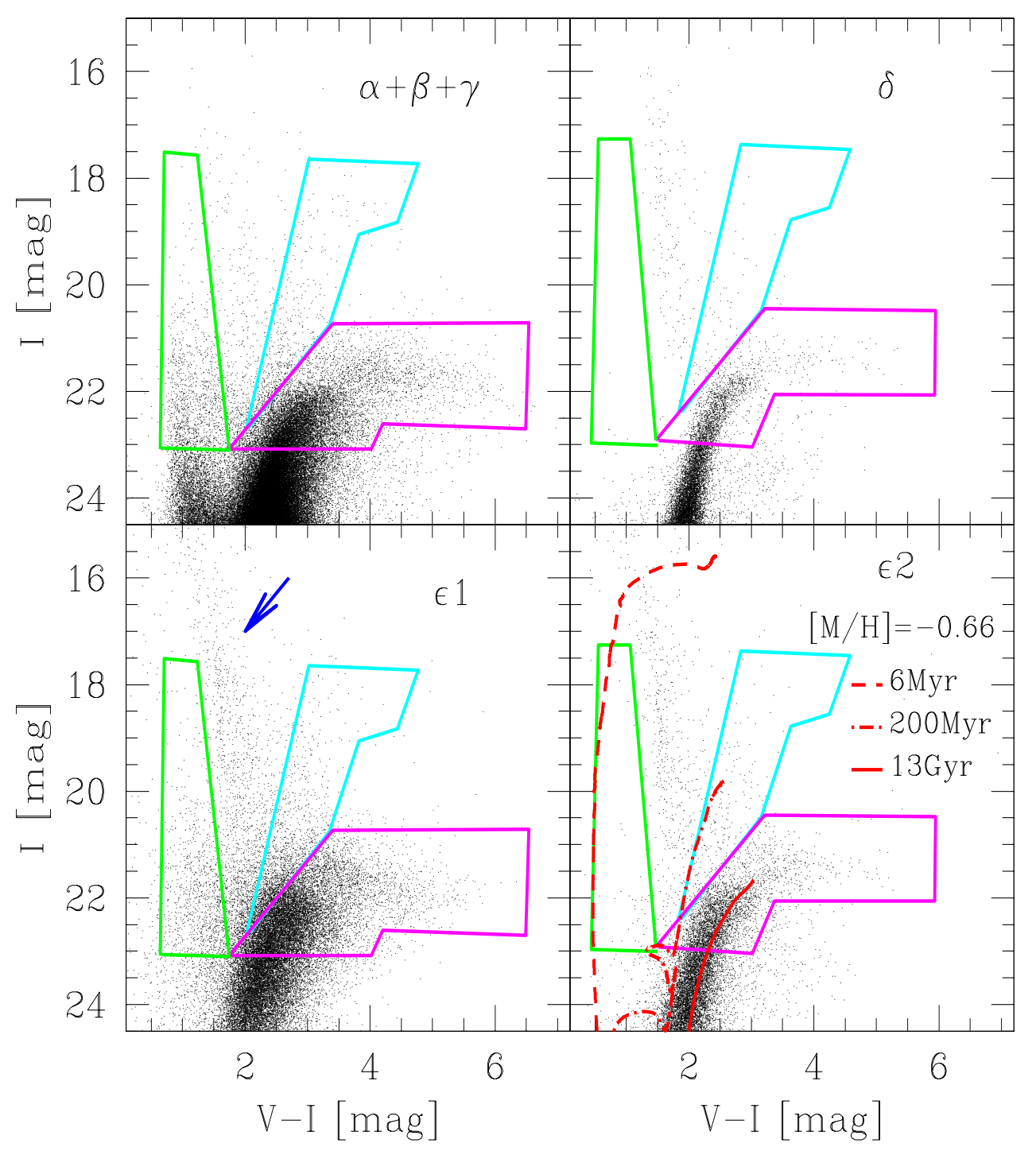

Fig. 4. - CMDs in I, V-I bands for the stars located within $6.5^{\prime}$ across the galaxy center. Top - space data of the pointings $\alpha, \beta$ and $\gamma($ left, $E(B-V)=0.78 \pm 0.06 \mathrm{mag}$ ) and $\delta$ (right, $E(B-V)=0.63 \pm 0.09 \mathrm{mag}$ ). The colored boxes mark the CMD regions adopted to select candidate IC10 stars. The green box includes young MS stars, the cyan box the intermediate-age stars and the pink one the old and intermediate-age RGs. The position of the boxes was changed according to the local reddening. Bottom - ground based data of the pointing $\epsilon(\epsilon 1, E(B-V)=0.78 \pm 0.10 ; \epsilon 2, E(B-V)=0.63 \pm 0.10 \mathrm{mag})$. The blue arrow marks the position of candidate field stars (left). The dashed and the dashed-dotted lines (right) display two young, scaled Solar isochrones ( $\mathrm{t}=6$, $200 \mathrm{Myr}$ ), while the solid line an old $\alpha$-enhanced isochrone ( $\mathrm{t}=13 \mathrm{Gyr})$. Young and old isochrones were constructed at fixed total metallicity $([\mathrm{M} / \mathrm{H}]=-0.66 \mathrm{dex})$.

luminosity over the entire body of the galaxy.

We are facing empirical evidence that dIs seem to show smaller $\mathrm{M} / \mathrm{L}$ ratios when compared with dwarf ellipticals (see Fig. 1 and Table 2 in Woo et al. 2008). The new data will allow us to constrain whether this evidence might be affected by observational biases. Moreover, they can shed new lights on the prediction that dwarf galaxies might have tidal radii significantly larger than empirical estimates (Havashi et al. 2003; Kazantzidis et al. 2004).

It is a real pleasure to thank the referee, Dr. I. Chilingarian, for his constructive suggestions.

\section{REFERENCES}

Bekki, K. 2008, MNRAS, 388,10

Benitez, N. 2000, ApJ, 536, 571

Capak, P. et al. 2004, AJ, 127, 180

Casertano, S. P. R., \& Shostak, G. S. 1980, A\&A, 81, 371

Castellani, V., Cignoni, M., Degl'Innocenti, S., Petroni, S., Prada Moroni, P. G. 2002, MNRAS, 334, 69
Cignoni, M., Degl'Innocenti, S., Prada Moroni, P. G., Shore, S. N. 2006, A\&A, 459, 783

Cohen, R. J. 1979, MNRAS, 187, 839

Demers, S., Battinelli, P., Letarte, B. 2004, A\&A, 424, 125

Ferguson, H. C. et al. 2004, ApJ, 600L, 107

Fukugita, M., Shimasaku, K., Ichikawa, T. 1995, PASP, 107, 945 
Girardi, L., Groenewegen, M. A. T., Hatziminaoglou, E., da Costa, L 2005, A\&A, 436, 895

Hayashi, E., Navarro, J. F., Taylor, J. E., Stadel, J., Quinn, T. 2003, ApJ, 584, 541

Huchtmeier, W. K. 1979, A\&A , 75, 170 (H79)

Huchtmeier, W. K., Richter, O. -G. 1988, A\&A , 203, 237

Hunter, D. A. 2001, ApJ, 559, 225

Jarrett, T. H. Chester, T., Cutri, R., Schneider, S. E., Huchra, J. P. 2003, AJ, 125, 525

Kazantzidis, S., Mayer, L., Mastopietro, C., Diemand, J., Stadel, J., Moore, B. 2004, ApJ, 608, 663

Kormendy, J., Fisher, D. B., Cornell, M. E., \& Bender, R. 2009, ApJS, 182, 216

Landolt, A.U., 1983, AJ, 88, 853

Landolt, A. U. 1992, AJ, 104, 340

Massey, P. \& Armandroff, T. E. 1995, AJ, 109, 2470

Mateo, M. 1998, ARA\&A, 36, 435

McConnachie, A. W., Huxor, A., Martin, N. F. et al. 2008, ApJ, 688, 1009

Pietrinferni, A., Cassisi, S., Salaris, M., Castelli, F. 2006, ApJ, 642, 797

Pietrinferni, A., Cassisi, S., Salaris, M., Castelli, F. 2004, ApJ, 612,168
Reyle, C., Marshall, D. J., Robin, A. C., Schultheis, M. 2009, A\&A, 495, 819

Richer, M. G. et al. 2001, A\&A, 370, 34

Sakai, S., Madore, B. F., Freedman, W. L. 1999, ApJ, 511, 671

Sanna, N. et al. 2008, ApJ, 688, L69

Sanna, N. et al. 2009, ApJ, 699, L84

Shostak, G. S., Skillman, E. D. 1989, 1989, A\&A, 214, 33 (SS89)

Sirianni, M. et al. 2005, PASP, 117, 1049

Stetson, P. B. 1994, PASP, 106, 250

Stetson, P.B, 1987, PASP, 99, 191

Tikhonov, N. A., Galazutdinova, O. A. 2009, AstL, 35, 748

Tolstoy, E., Hill, V., \& Tosi, M. 2009, ARA\&A, 47, 371

Vacca, W. D.; Sheehy, C. D., Graham, J. R. 2007, ApJ, 662, 272 van den Bergh, S. 2000, The Galaxies of the Local Group, ed.

Cambridge Astrophysical Series

Wang, Q. D., Whitalker, K. E., Williams, R. 2005, MNRAS, 362, 1065

Wilcots, E. M., Miller, B. W. 1998, AJ, 116, 2363

Woo, J., Courteau, S., Dekel, A. 2008, MNRAS, 390, 1453

Wyse, R. F. G. 2010, AN, 331, 526 